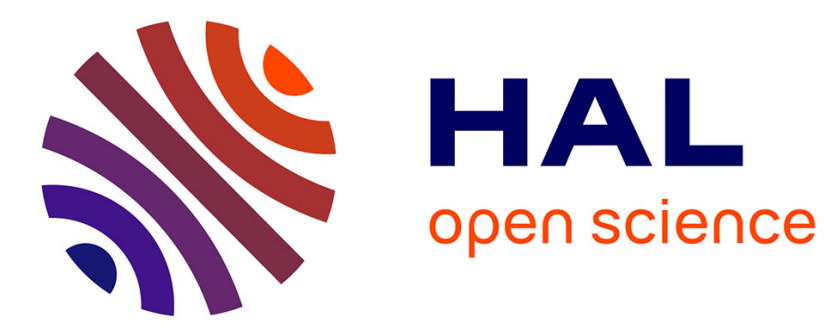

\title{
Convolutive Bottleneck Network with Dropout for Dysarthric Speech Recognition
}

\author{
Toru Nakashika, Toshiya Yoshioka, Tetsuya Takiguchi, Yasuo Ariki, Stefan \\ Duffner, Christophe Garcia
}

\section{- To cite this version:}

Toru Nakashika, Toshiya Yoshioka, Tetsuya Takiguchi, Yasuo Ariki, Stefan Duffner, et al.. Convolutive Bottleneck Network with Dropout for Dysarthric Speech Recognition. Transactions on Machine Learning and Artificial Intelligence, 2014, 2, 2, pp.1-15. 10.14738/tmlai.22.150 . hal-01301040

\section{HAL Id: hal-01301040 \\ https://hal.science/hal-01301040}

Submitted on 27 Mar 2017

HAL is a multi-disciplinary open access archive for the deposit and dissemination of scientific research documents, whether they are published or not. The documents may come from teaching and research institutions in France or abroad, or from public or private research centers.
L'archive ouverte pluridisciplinaire HAL, est destinée au dépôt et à la diffusion de documents scientifiques de niveau recherche, publiés ou non, émanant des établissements d'enseignement et de recherche français ou étrangers, des laboratoires publics ou privés. 


\title{
Convolutive Bottleneck Network with Dropout for Dysarthric Speech Recognition
}

\author{
Toru Nakashika ${ }^{1}$, Toshiya Yoshioka ${ }^{1}$, Tetsuya Takiguchi ${ }^{1}$, Yasuo Ariki $^{1}$, \\ Stefan Duffner ${ }^{2}$, Christophe Garcia ${ }^{2}$ \\ ${ }^{1}$ Department of System Informatics, Kobe University, JAPAN \\ ${ }^{2}$ Université de Lyon, CNRS, INSA-Lyon, LIRIS, UMR 5205, FRANCE \\ 1 nakashika@me.cs.scitec.kobe-u.ac.jp
}

\begin{abstract}
In this paper, we investigate the recognition of speech produced by a person with an articulation disorder resulting from athetoid cerebral palsy. The articulation of the first spoken words tends to become unstable due to strain on speech muscles, and that causes degradation of speech recognition. Therefore, we propose a robust feature extraction method using a convolutive bottleneck network (CBN) instead of the well-known MFCC. The CBN stacks multiple various types of layers, such as a convolution layer, a subsampling layer, and a bottleneck layer, forming a deep network. Applying the CBN to feature extraction for dysarthric speech, we expect that the CBN will reduce the influence of the unstable speaking style caused by the athetoid symptoms. Furthermore, we also adopt dropout in the output layer since automatically-assigned labels to the dysarthric speech are usually unreliable due to ambiguous phonemes uttered by the person with speech disorders. We confirmed its effectiveness through word-recognition experiments, where the CNN-based feature extraction method outperformed the conventional feature extraction method.
\end{abstract}

Keywords: Articulation disorders, Feature extraction, Convolutional neural network, Bottleneck feature, Dropout, Dysarthric speech.

\section{INTRODUCTION}

Recently, the importance of information technology in the welfare-related fields has increased. For example, sign language recognition using image recognition technology [1], text reading systems from natural scene images [2], and the design of wearable speech synthesizers for voice disorders $[3,4]$ have been studied.

As for speech recognition technology, the opportunities in various environments and situations have increased (e.g., operation of a car navigation system, lecture transcription during meetings, etc.). However, degradation can be observed in the case of children [5], 
persons with a speech impediment, and so on, and there has been very little research on orallychallenged people, such as those with speech impediments. It is hoped that speech recognition systems will one day be able to recognize their voices.

One of the causes of speech impediments is cerebral palsy. Cerebral palsy results from damage to the central nervous system, and the damage causes movement disorders. Three general times are given for the onset of the disorder: before birth, at the time of delivery, and after birth. Cerebral palsy is classified as follows: 1 ) spastic type 2) athetoid type 3) ataxic type 4) atonic type 5) rigid type, and a mixture of types [6].

In this paper, we focused on a person with an articulation disorder resulting from the athetoid type of cerebral palsy as in [7]. Athetoid symptoms develop in about $10-15 \%$ of cerebral palsy sufferers. In the case of a person with this type of articulation disorder, the first movements are sometimes more unstable than usual. That means, the case of movements related to speaking, the first utterance is often unstable or unclear due to the athetoid symptoms. Therefore, we recorded speech data for a person with a speech impediment who uttered a given word several times, and we investigated the influence of the unstable speaking style caused by the athetoid symptoms.

In speech recognition technology, frame-wise features such as mel-frequency cepstral coefficients (MFCC), linear predictive coding (LPC), and an autoregressive model (AR) have been widely used so far. However, these features do not capture the temporal information unless delta features are used. Especially for dysarthric speech, where the signals fluctuate more obviously than the signals uttered by a physically unimpaired person, spectral transition in the short term is considered to be an important factor in capturing the local temporal-dimensional characteristics. In this paper, we employ a convolutional neural network (CNN) [8, 9])-based approach to extract disorder- dependent features from a segment MFCC map. The CNN is regarded as a successful tool and has been widely used in recent years for various tasks, such as image analysis [10,11,12], a spoken language [13], and music recognition [14]. A CNN consists of a pipeline of convolution and pooling operations followed by a multi-layer perceptron. In dysarthric speech, the key points in time-spectral local areas of an input feature map are often shifted slightly due to the fluctuation of the speech uttered by a person with an articulation disorder. However, thanks to the convolution and pooling operations, we can train the CNN robustly to deal with the small local fluctuations. Furthermore, we expect that the CNN extracts specific features associated with the articulation disorder we are targeting when we train the network using only the speech data of the articulation disorder.

For the research described in this paper, we used a convolutive bottleneck network (CBN) [15], which is an extension of a CNN, to extract disorder-specific features. A CBN stacks a bottleneck layer, where the number of units is extremely small compared with the adjacent layers, following the CNN layers. Due to the bottleneck layer having a small number of units, it 
is expected that it can aggregate the propagated information and extract fundamental features included in an input map. Furthermore, we also use a dropout technique [16] to prevent overfitting appeared in the output layer. Since the label data (teaching signal) is obtained by forcedalignment from dysarthric speech where each phoneme fluctuates largely, the teaching signal for the training of the network is very unreliable. The dropout in the output layer ignores some information in the teaching signal, and thus will deal with the problem of the wrong alignment.

This paper is organized as follows: we briefly review the fundamental method, CNN in Section 2. The proposed feature extraction method and the structure of the CBN used in the experiments are presented in Section 3. In Section 4, we show the experimental results, and we give our conclusions regarding in Section 5.

\section{CONVOLUTIONAL NEURAL NETWORK}

In this section, we describe a convolution layer and a pooling layer, which are fundamental components of a CNN (convolutional neural network).

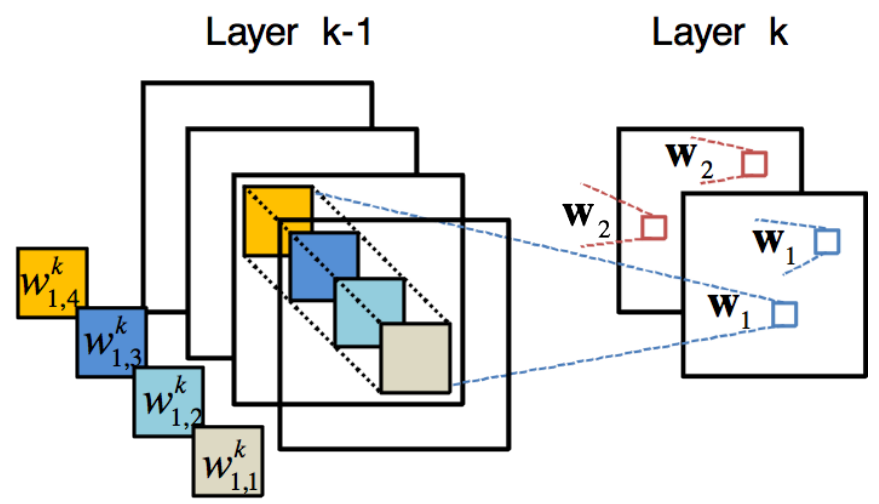

Figure 1: Feature maps in a convolutional layer (right) feed input feature maps (left)

\subsubsection{Convolution layer}

Assuming that we have a two-dimensional input feature map $x \in \mathbb{R}_{n}^{N_{n}^{z} \times N_{m}^{z}}$ and a convolutive filter $w \in \mathbb{R}^{N_{*}^{*} \times N_{m}^{w}}$, the output of a convolutive operation $h=x * w$ also becomes a two-dimensional feature with the size of $N_{n}^{h} \times N_{m}^{h}\left(N_{n}^{h} \equiv N_{n}^{z}-N_{n}^{w}+1\right.$ and vice versa). A CNN generally has a number of such filters $\left\{\boldsymbol{w}_{1}, \cdots, \boldsymbol{w}_{L}\right\}$ in a convolutive layer, and feeds an input $\boldsymbol{x}$ using each filter to create the corresponding outputs $\left\{h_{1}, \cdots, h_{L}\right\}$, which is referred to as a feature map.

Given all of the feature maps in the $(k-1)$ th layer $\left\{h_{1}^{k-1}, \cdots, h_{i}^{k-1}, \cdots, h_{I}^{k-1}\right\}$, the $j$ th feature map $h_{j}^{k} \in \mathbb{R}^{N_{k} \times N_{h}}$ in the $k$ th (convolution) layer can be calculated as 


$$
h_{j}^{k}=f\left(\sum_{i \in I} w_{j, i}^{k} \times h_{i}^{k-1}+b_{j}^{k}\right) \text {, }
$$

where $w_{j, i}^{k}$ and $b_{j}^{k}$ indicate a predictable filter from the $i$ th feature map in $(k-1)$ th layer to the $j$ th map in the $k$ th layer and a bias map of the $j$ th map in the $k$ th layer, respectively. In this paper, we used an element-wise sigmoid function for the activation function $f$ as follows

$$
f(\boldsymbol{x})=\frac{1}{1+e^{-\boldsymbol{x}}},
$$

where the fraction bar indicates element-wise division.

Each unit in a convolution layer is connected to the units in the corresponding local area of size $N_{n}^{w} \times N_{m}^{w}$ in the previous layer (local receptive field). In other words, the convolution layer in a CNN captures local patterns in an input map using various filters (Figure 1).

\subsubsection{Pooling layer}

Followed by the convolution layer, a pooling procedure is generally used in a CNN, creating what is called a pooling layer. Each unit in the pooling layer aggregates responses in the local subregion $\mathcal{P}(M \times M)$ in the previous convolution layer. As a result, a feature map in the pooling layer has the size of $N_{n}^{h} / M \times N_{m}^{h} / M$.

There exist various pooling methods (e.g. max-pooling), but we use average-pooling in this paper, calculated as follows

$$
h_{j}^{k+1}=f\left(w_{j}^{k+1} \cdot \frac{1}{M^{2}} \sum_{(u, v) \in \mathcal{P}} h_{j, u, v}^{k}+b_{j}^{k+1}\right),
$$

where $w_{j}^{k+1}$ and $b_{j}^{k+1}$ are weight parameter and a bias parameter of the $i$ th feature map in the pooling layer ( $(k-1)$ th layer), respectively. ${ }^{k}{ }_{j, u, v}$ represents the unit in the corresponding subregion identified with $(u, v)$ in the feature map in the $k$ th layer.

This pooling process enables the network to ignore small position shifts of a key point in the input feature map since it aggregates information in the local area. 


\section{PROPOSED METHOD}

In our approach, we use a convolutional neural network (CNN) that has a bottleneck layer in the network, referred to as a convolutive bottleneck network (CBN) [15], for capturing speakerdependent features from a dysarthric speech signal.

\subsection{Convolutive Bottleneck Network}

A CBN consists of an input layer, convolution layer and pooling layer pairs, fully-connected MLPs (multi-layer perceptrons) with a bottleneck structure, and an output layer in the order shown in Figure 2. In our approach, the CBN feeds a mel map (two-dimensional acoustic features in time-mel-frequency) and outputs 54 phoneme labels. The MLP shown in Figure 2 stacks three layers ( $\mathrm{m} 1, \mathrm{~m} 2$, m3 ), where we give 108 units, 30 bottleneck units, and 108 units in each layer, respectively. The filter sizes in the convolution layer and the pooling layer will be discussed in the experimental section. Since the bottleneck layer has reduced the number of units for the adjacent layers, we can expect that each unit in the bottleneck layer aggregates information and behaves as a compact feature descriptor that represents an input with a small number of bases, similar to other feature descriptors, such as MFCC, LDA (linear discriminant analysis) or PCA (principal component analysis). It is reported that a feature extraction method using bottleneck features improved speech recognition accuracy from a well-known ABN (American Broad News) corpus [17], and in this paper as well, we use such features (the unit values in the bottleneck layer) for speech recognition, instead of using MFCC. The extracted features are obtained from the statistically-trained speaker-dependent CBN; hence, it is expected that it better represents characteristics in the speech of the target articulation disordered speech than MFCC does.

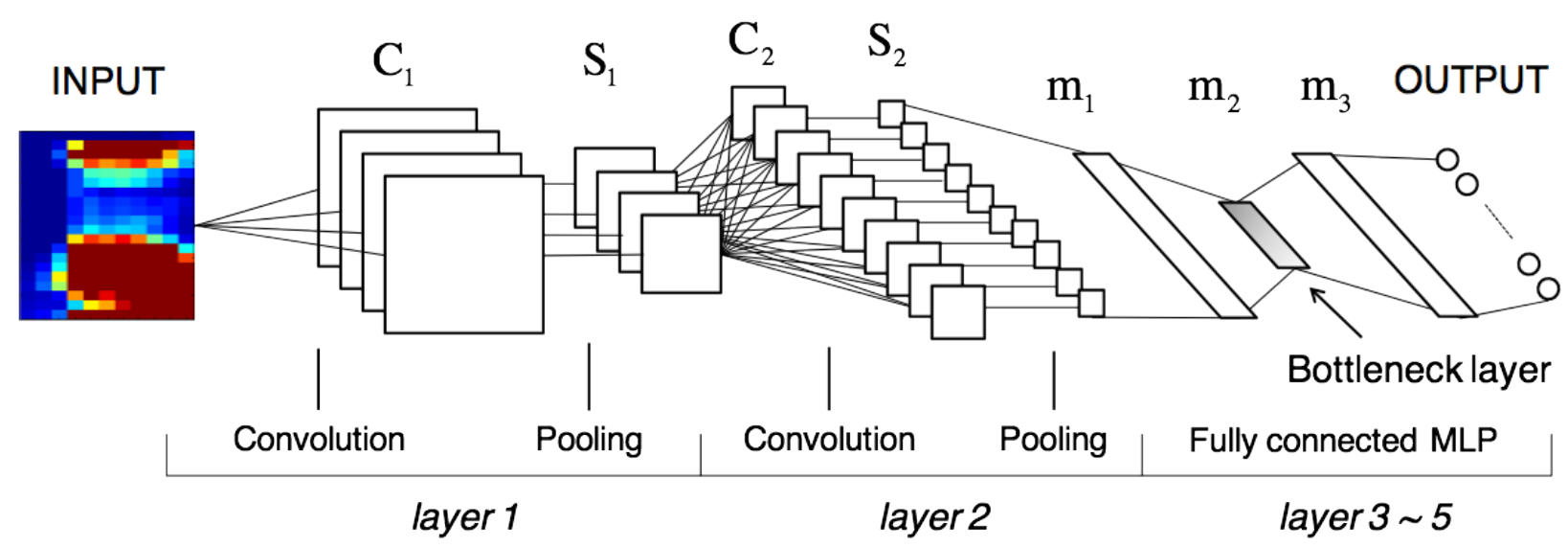

Figure 2: Convolutive Bottleneck Network (CBN) 


\subsection{Bottleneck feature extraction from dysarthric speech}

Figure 3 shows a system flowchart of our method, where speaker-specific features are extracted using a CBN. First, we prepare the input features for training a CBN from a speech signal uttered by person with an articulation disorder. After calculating short-term mel spectra from the signal, we obtain mel maps by dividing the mel spectra into segments with several frames (13 frames in our experiments) allowing overlaps. For the output units of the CBN, we use phoneme labels that correspond to the input mel-map. For example, when we have a mel map with the label /i/, only the unit corresponding to the label /i/ is set to 1 , and the others are set to 0 in the output layer. The label data is obtained by forced alignment using a hidden Marcov model (HMM) from the speech data. The parameters of the CBN are trained by backpropagation with stochastic gradient descent, starting from random values. The bottleneck (BN) features in the trained $\mathrm{CBN}$ are then used in the training of another HMM for speech recognition.

In the test stage ("Feature extraction" in Figure 3), we extract features using the CBN, which feeds the mel maps obtained from test data and tries to produce the appropriate phoneme labels in the output layer. Again, note that we do not use the output (estimated) labels for the following procedure, but we use the BN features in the middle layer, where it is considered that information in the input data is aggregated. Finally, the system recognizes dysarthric speech by feeding the extracted BN features into HMMs.

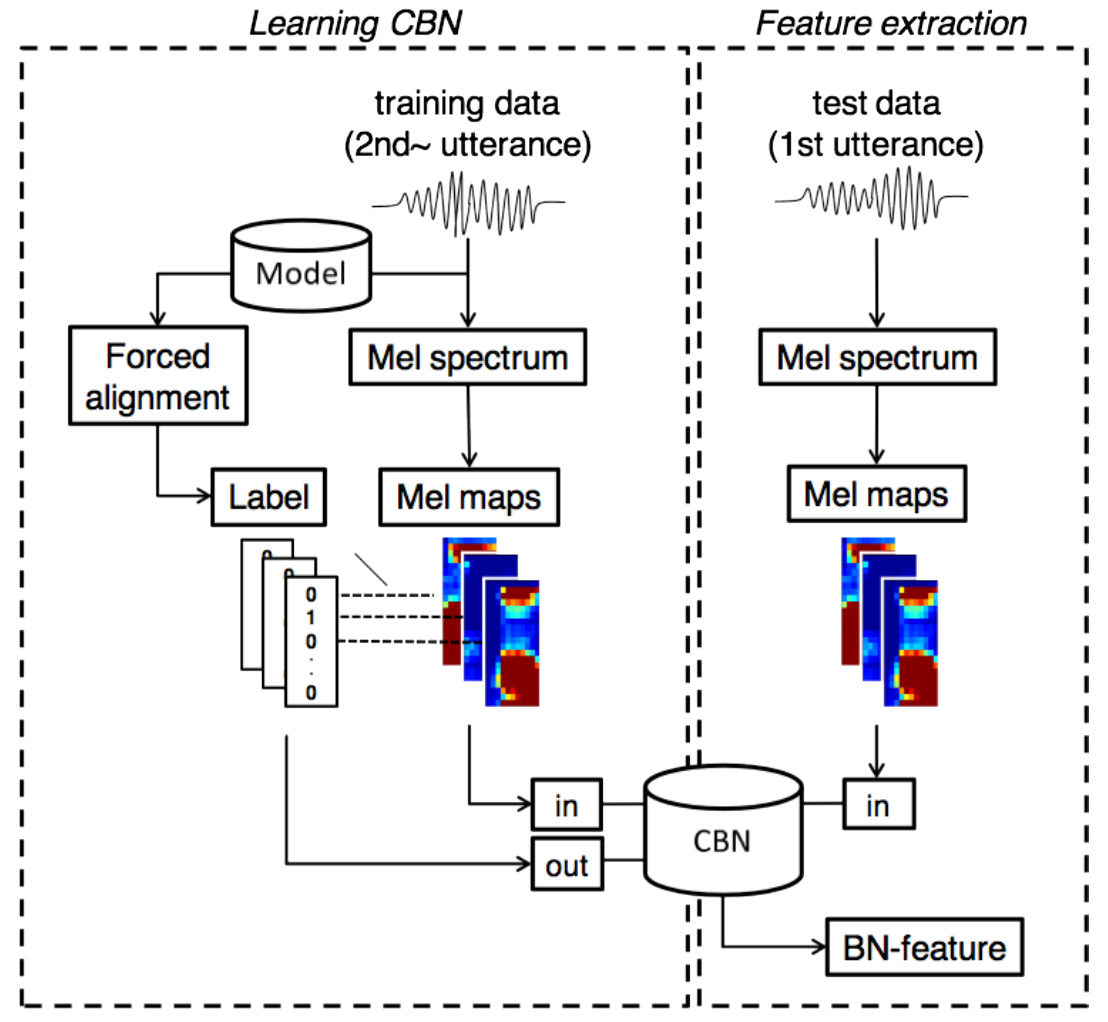

Figure 3: Flow of our feature extraction method for dysarthric speech using a convolutive bottleneck network 


\section{EXPERIMENTAL EVALUATION}

\subsection{Word recognition experiments (speaker A)}

In this section, we discuss and evaluate our experiments of word recognition using a CBN presented in the previous section. Since we want to investigate the effectiveness of the CBN, we did not use dropout in the output layer of the CBN in these experiments.

\subsubsection{Conditions}

Our feature extraction method was evaluated on word recognition tasks for one male person (identified with "speaker A") with an articulation disorder. We recorded 216 words included in the ATR Japanese speech database [18] repeating each word five times (Figure 4). The utterance signal was sampled at $16 \mathrm{kHz}$ and windowed with a 25-msec Hamming window every $10 \mathrm{msec}$. Then we clipped each utterance manually. In our experiments, the first utterances of each word were used for evaluation, and the other utterances (the 2nd through 5th utterances) were used for the training of both a CBN and an acoustic model. We used the HMMs (54 context-independent phonemes) with 5 states and 8 mixtures of Gaussians for the acoustic model.

We trained and evaluated three CBNs: 28 units, 30 units, and 32 units in the bottleneck (BN) layer. The extracted BN features from each CBN were compared with 28-, 30-, and 32dimensional MFCC+ $\triangle M F C C$, respectively.

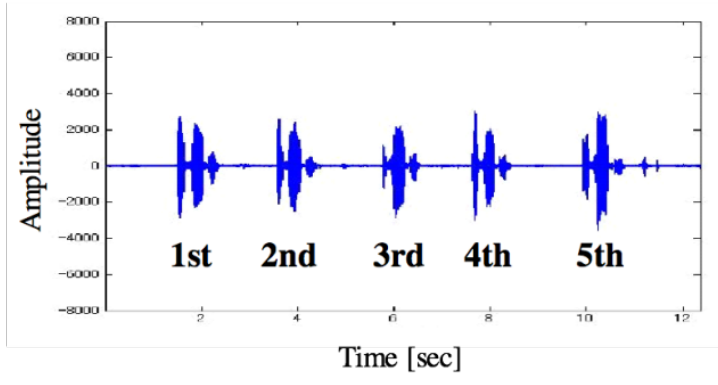

Figure 2: Example of recorded speech data

\subsubsection{Training of CBN}

In this section, we explain the training conditions of the $\mathrm{CBN}$ in detail. We trained the network using pairs of a mel map from the 2nd through 5th utterances and the label, as shown in Figure 3.

Each value of the convolutive filter $w_{j, i}^{k}$ (and the weight $w_{j}^{k+1}$ ) is initialized by [19] as follows:

$$
w_{j, i}^{k} \ni w \sim \mathcal{U}\left(-\sqrt{\frac{6}{N_{j}+N_{j+1}}}, \sqrt{\frac{6}{N_{j}+N_{j+1}}}\right),
$$


where $\mathcal{U}(a, b)$ denotes uniform distribution at the interval from $a$ to $b . N_{j}$ and $N_{j+1}$ indicate the numbers of input dimensions and output dimensions at the $j$ th layer, respectively. The bias parameters $b_{j}^{k}$ and $b_{i}^{k+1}$ were set to 0 as initial values.

These parameters were trained so as to minimize the errors between the target labels and the output values using a back-propagation algorithm. We iterated batch-based training with 50 frames in a mini-batch 100 times, with a fixed learning rate of 0.1 .

\subsubsection{CBN architectures}

In this section, we discuss our preliminary experiment in which we change the architecture of a CBN (such as the number of feature maps and the size of a convolution filter) as shown in Table 1. In this preliminary experiment, we will see which architecture produced the best recognition accuracy. All the architectures have two pairs of a convolution layer and a pooling layer followed by three-layer MLPs with a bottleneck layer, forming a nine-layer network in total. We used 108, 30 and 108 units in the MLP part, in this order, for all the architectures (the bottleneck feature had 30 dimensions in this preliminary experiment). For the input layer, we used a 39-dimensional mel map with 13 frames without overlapping. The size of the map was $39 \times 13$. When we use 'Arc1' in Table 1, for example, the feature maps in each convolution and pooling layer have $36 \times 12,12 \times 4,9 \times 3$, and $3 \times 1$ sizes, in this order. In this case, $3 \times 27(=81)$ units are fully connected to the first layer of the MLP part.

Table 2 shows recognition accuracies obtained from each architecture. As shown in Table 2, we obtained the best word recognition accuracies from 'Arc1', although it did not always outperform the other architectures with respect to the MSE and label classification accuracy. This is considered to be due to the fact that the extracted bottleneck features of 'Arc1' were more abstract and suited to the acoustic model in word recognition. For the remaining experiments, we used 'Arc1' for the CBN architecture except for the number of units in the bottleneck layer.

Table 1: Filter size and number of feature maps for each architecture. The values for C1 (and C2) indicate filter size of the first (and the second) convolution layer that has \#1 maps (and \#2 maps), respectively. Each convolution layer is associated with the pooling layer (S1 and S2). The values for S1 and S2 mean the pooling factor M)

\begin{tabular}{|r|c|c|c|c|c|c|}
\hline & C1 & S1 & \#1 & C2 & S2 & \#2 \\
\hline Arc1 & $4 \times 2$ & 3 & 13 & $4 \times 2$ & 3 & 27 \\
\hline Arc2 & $10 \times 4$ & 2 & 13 & $10 \times 4$ & 2 & 27 \\
\hline Arc3 & $4 \times 2$ & 3 & 18 & $4 \times 2$ & 3 & 36 \\
\hline Arc4 & $4 \times 2$ & 4 & 13 & $4 \times 2$ & 2 & 27 \\
\hline
\end{tabular}




\begin{tabular}{|r|r|r|r|r|r|r|}
\hline Arc5 & $8 \times 6$ & 2 & 13 & $8 \times 2$ & 3 & 27 \\
\hline
\end{tabular}

Table 2: Word recognition accuracies using the bottleneck features (Word-Acc.) obtained from each architecture, along with the mean squared error of the closed data (MSE), and the open classification accuracies of the phoneme labels using a CBN only (without using the acoustic models) (Phoneme-Acc.)

\begin{tabular}{|l|r|r|r|r|r|}
\hline & Arc1 & Arc2 & Arc3 & Arc4 & Arc5 \\
\hline MSE $\left(\times 10^{-1}\right)$ & 2.42 & 2.01 & 2.32 & 2.43 & 2.09 \\
\hline Phoneme-Acc. (\%) & 48.7 & 49.3 & 47.9 & 49.4 & 49.5 \\
\hline Word-Acc. (\%) & $\mathbf{8 8 . 0}$ & 87.7 & 82.4 & 84.3 & 83.8 \\
\hline
\end{tabular}

\subsubsection{Results and discussion}

Figure 5 shows the word recognition accuracies comparing our CBN features with the conventional MFCC features, when changing the number of feature dimensions. As shown in Figure 5, the use of bottleneck features in a convolutive network improved the accuracies from $84.3 \%$ to $88.0 \%$ (30 dimensions). This is due to the robustness of the CBN features to small local fluctuations in a time-melfrequency map, caused by the dysarthric speech.

We further investigated our method to check the effectiveness of the convolution layer (and pooling layer). In this evaluation, we replaced some convolution layers (here we refer to the pair of a convolution layer and a pooling layer as simply a "convolution layer") with fullyconnected layers in the network ('Arc1'). First, we replaced the second convolution layer ('Layer $2^{\prime}$ in Figure 2) with a fully-connected layer with 108 units. Second, we alternated two convolution layers with two fully-connected layers with 108 units, which is regarded as a 7-layer DBN (deep bottleneck network). The results are summarized in Table 3. From Table 3, we notice that the more convolution layers the network had, the better the performance of the system. Again, we consider that this is because the convolution filter captured characteristics in the input maps, making it robust to local fluctuations. When we compare the fully-connected model (DBN) with the MFCC, we see that the DBN performs poorly since it tends to suffer from over-fitting and a lack of robustness to the open data, especially in dysarthric speech, which fluctuates every time the speaker begins speaking. 


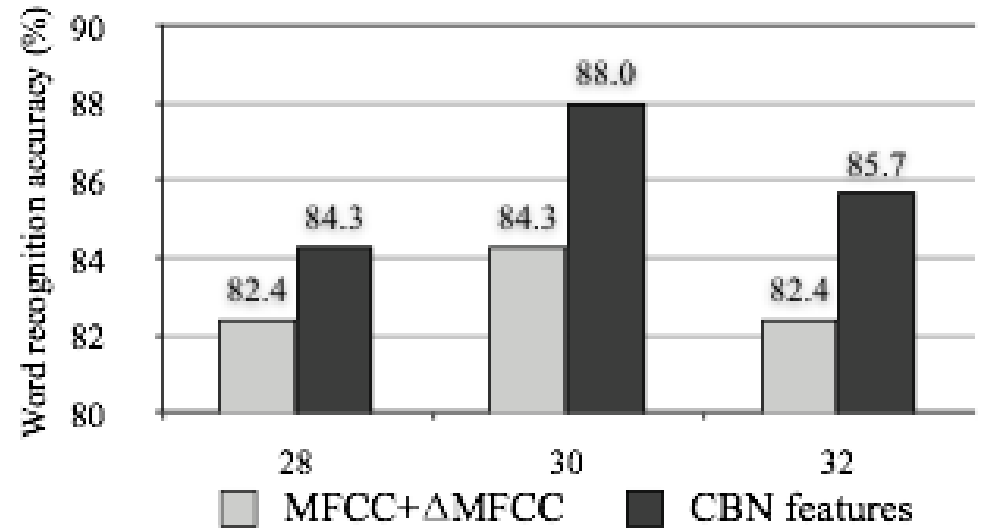

Figure 5: Word recognition accuracies (\%) for the utterances of "speaker A" using bottleneck features in a convolutional network

Table 3: Word recognition accuracies as the number of convolution layers changed.

\begin{tabular}{|c|c|}
\hline \# of conv v.s. full layers & Acc. (\%) \\
\hline No conv, 5 full (DBN) & 83.3 \\
\hline 1 conv, 4 full (CBN) & 84.7 \\
\hline 2 conv, 3 full (CBN) & 88.0 \\
\hline Baseline (MFCCs) & 84.3 \\
\hline
\end{tabular}

\subsection{Word recognition experiments (speaker B)}

We confirmed improvement of recognition accuracies by using a CBN feature extraction method in the previous experiments. However, the symptom of the articulation disorder and the tendency of the fluctuation in dysarthric speech vary on the speaker. In this section, we show experimental results using speech uttered by another person (female; "speaker B") with an articulation disorder.

\subsubsection{Conditions}

We conducted word recognition experiments similarly to the previous experiments using speech of words included in the ATR Japanese speech database, uttered by "speaker B". The speech data consists of 200 words, each of which was repeated three times (600 word speech in total). In the experiments, the first utterances of each word (200 words) were used for the test, and the other utterances ( 400 words) were used for the training of a CBN and acoustic models. The other configurations were set same as the experiments with "speaker $A$ ". 


\subsubsection{Results and discussion}

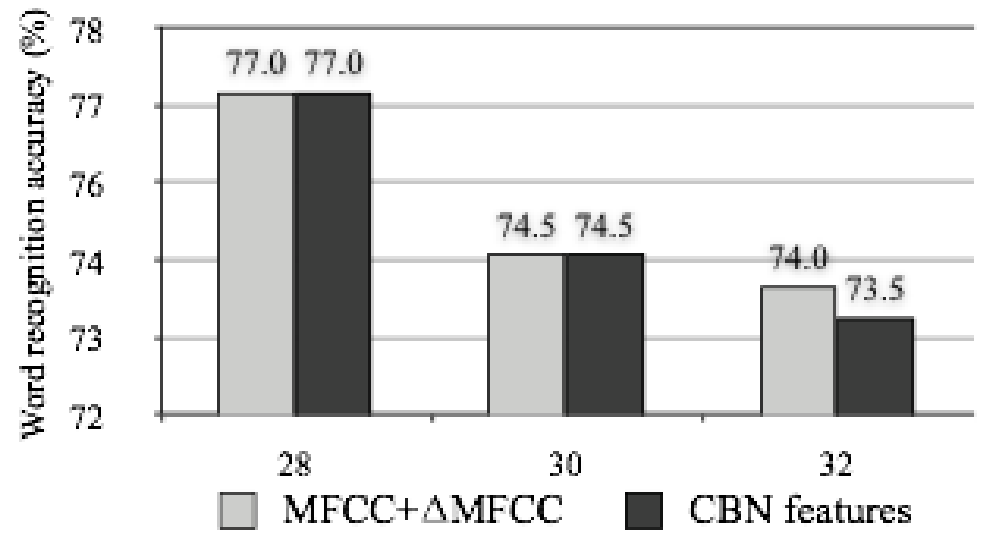

Figure 6: Word recognition accuracies (\%) for the utterances of "speaker B" using bottleneck features in a convolutional network

Figure 6 shows experimental results of "speaker B". As shown in Figure 6, unlike the results with "speaker A", we did not see improvements of recognition accuracy with CBN features. One of the reasons is considered to be due to the assigned phoneme labels included in the training data. In other words, there exist gaps between the estimated labels obtained by forced alignment and actual labels, because the speaker could not utter some words correctly. It means that we give incorrect teaching signals to the network, which degrades the performance in the training. According to the experimental results, this problem arises especially on the second speaker. Figures 7 and 8 show examples of alignment for "speaker A" and "speaker B", respectively. Each figure compares the results of forced-alignment obtained by using HMMs (a) and manually-assigned actual labels (b). As shown in Figure 7, the forced-alignment result of "speaker A" was similar to the actual labels. However, in case of "speaker B", the segment for phoneme $/ \mathrm{y} /$ was enlarged and the following phoneme bounds were quite wrong as shown in Figure 8 . This means that the features that should correspond to the phoneme $/ \mathrm{a} / \mathrm{or} / \mathrm{m} /$ were regarded as the phoneme $/ y /$ in the training. 
(a) Forced -alignment

(b) Actual

(a) Forced -alignment

(b) Actual

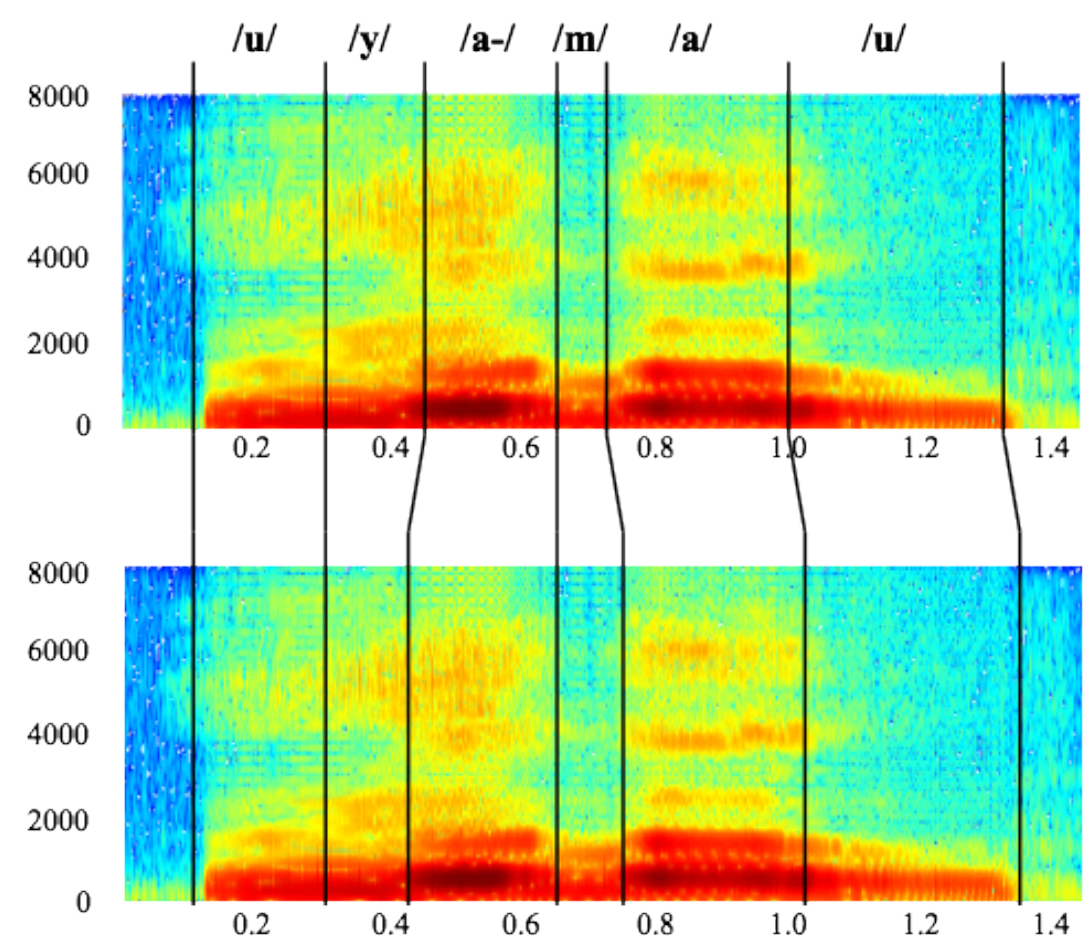

Figure 7: Example of forced-alignment for "speaker A"

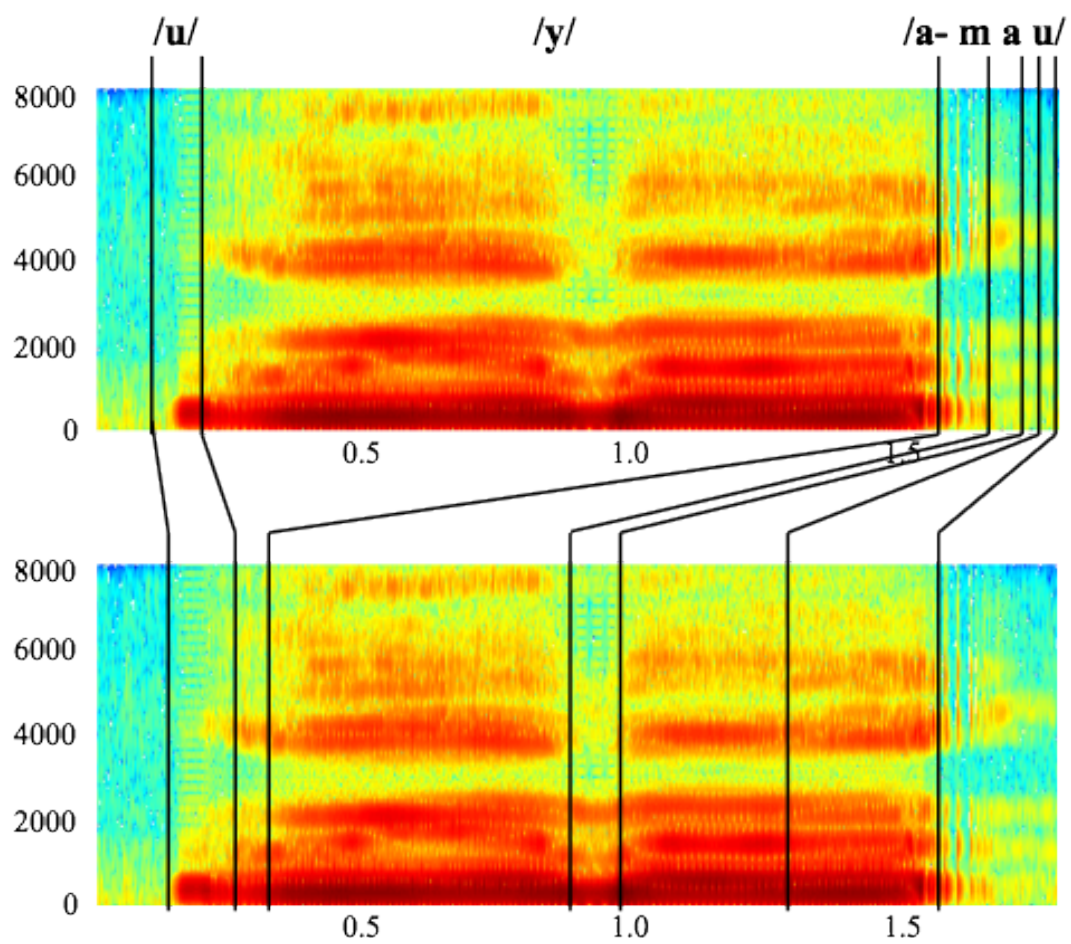

Figure 8: Example of forced-alignment for "speaker A" 


\subsubsection{Dropout effect}

To solve the problem discussed in the previous subsection, we employ a dropout technique [16] when training the $\mathrm{CBN}$, in this paper. The dropout gives the network generalization capabilities by ignoring some randomly-selected units (setting the weights to zero) in a layer. We expect that the network with dropout in the output layer (training signal) prevents overfitting to the label data that is unreliable because of forced-alignment. Specifically, given an input vector $x_{p}$, the output of a network $f_{\text {out }}\left(x_{p}\right)$ is obtained by applying a binary mask as follows:

$$
f_{\text {out }}^{\prime}\left(x_{p}\right)=f_{\text {out }}\left(x_{p}\right) \circ m
$$

where $\circ$ denotes element-wise product, and each value of $m$ is randomly set to 1 with a probability $p_{d}\left(0\right.$ with a probability $\left.1-p_{d}\right)$. When each unit in the output layer randomly takes the value of 0 even when the value is activated, the network tries to capture abstracts hidden in the data. In our experiments, we used $p_{d}=0.5$.

Figure 9 shows experimental results, adding the result of a CBN with dropout from Figure 6. As shown in Figure 9, the accuracy was highly improved when we apply dropout. Compared with the baseline (MFCC+ $\mathrm{MFCC}$ ), the dropout method (CBN+DR) achieved an up-to-5-point improvement. Through these experiments, "ambiguous" training using dropout worked well especially for the speech where phonemes fluctuate largely and it is difficult to prepare the exact labels, as the speech uttered by "speaker B".

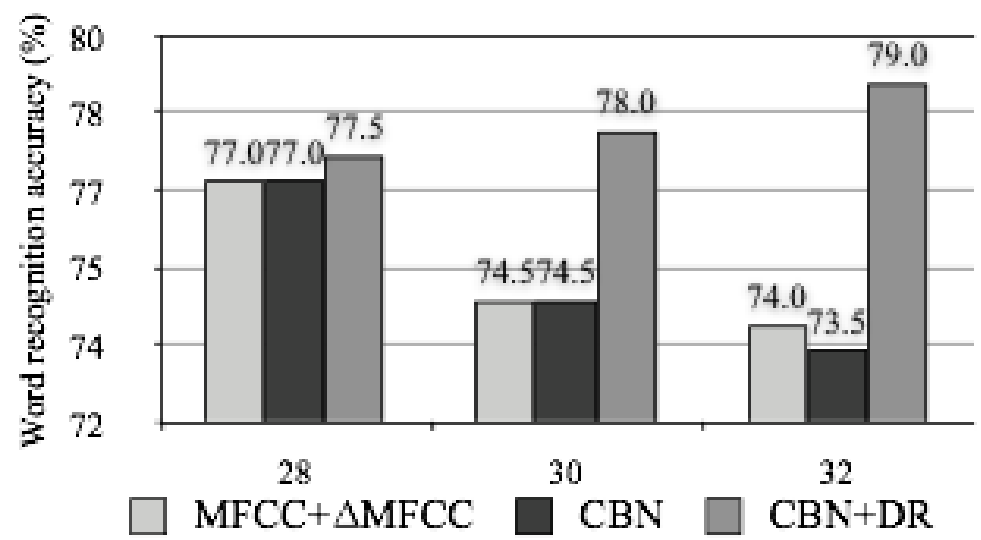

Figure 9: Word recognition accuracies (\%) for the utterances of "speaker B" using bottleneck features and dropout (DR)

\section{CONCLUSIONS}

The articulation of speech uttered by persons with speech disorders tends to become unstable due to strain on their speech-related muscles. This paper described a robust feature 
extraction method using a convolutive bottleneck network (CBN). In word recognition experiments, our method achieved an approximately 4-point improvement compared with the conventional MFCC features for a male speaker ("speaker A"). For the another speaker ("speaker B"), we could not obtain better performance from the same technique as "speaker $A^{\prime \prime}$, because the speech fluctuates largely and it was difficult to estimate boundaries of phonemes correctly by forced-alignment. However, when we realize ambiguous training using dropout, our method achieved a 5-point improvement compared with MFCC features for "speaker B".

Since the tendency of the fluctuation in dysarthric speech depends on the speaker, we would like to apply and investigate our method to more persons with speech disorders in the future.

\section{REFERENCES}

[1]. S. Cox and S. Dasmahapatra, "High-Level Approaches to Confidence Estimation in Speech Recognition," IEEE Trans. on SAP, vol. 10, No. 7, pp. 460-471, 2002.

[2]. M. K. Bashar, T. Matsumoto, Y. Takeuchi, H. Kudo and N. Ohnishi, "Unsupervised Texture Segmentation via Wavelet-based Locally Orderless Images (WLOIS) and SOM," 6th IASTED International Conference COMPUTER GRAPHICS AND IMAGING, 2003.

[3]. T. Ohsuga and Y. Horiuchi and A. Ichikawa, "Estimating Syntactic Structure from Prosody in Japanese Speech," IEICE Trans. on Information and Systems, 86(3), pp. 558-564, 2003.

[4]. K. Nakamura and T. Toda and H. Saruwatari and K. Shikano, "Speaking Aid System for Total Laryngectomees Using Voice Conversion of Body Transmitted Artificial Speech," Interspeech 2006, pp. 1395-1398, 2006.

[5]. D. Giuliani and M. Gerosa, "Investigating recognition of children's speech," ICASSP2003, pp. 137-140, 2003.

[6]. S. T. Canale and W. C. Campbell, "Campbell 's Operative Orthopaedics," Mosby-Year Book, 2002.

[7]. H. Matsumasa, T. Takiguchi, Y. Ariki, I. LI, T. Nakabayashi, "Integration of Metamodel and Acoustic Model for Speech Recognition," Interspeech 2008, pp.2234-2237, 2008.

[8]. Y. Lecun et al., “Gradient-based learning applied to document recognition," Proc. IEEE, pp. 2278-2324, 1998.

[9]. H. Lee et al., "Unsupervised feature learning for audio classification using convolutional deep belief networks," in Advances in Neural Information Processing Systems 22, pp. 1096-1104, 2004.

[10]. C. Garcia and M. Delakis, "Convolutional Face Finder: A Neural Architecture for Fast and Robust Face Detection," Pattern Analysis and Machine Intelligence, 2004.

[11]. M. Delakis and C. Garcia, "Text detection with Convolutional Neural Networks," Proc. of the Int. Conf. on Computer Vision Theory and Applications, 2008. 
[12]. R. Hadsell et el., "Learning long-range vision for autonomous off-road driving," Journal of Field Robotics, 2009.

[13]. G. Montavon, "Deep learning for spoken language identification," NIPS Workshop on Deep Learning for Speech Recognition and Related Applications, 2009.

[14]. T. Nakashika, C. Garcia, and T. Takiguchi, "Local-feature-map Integration Using Convolutional Neural Networks for Music Genre Classication," Interspeech 2012, 2012.

[15]. K. Vesely et al. "Convolutive bottleneck network features for LVCSR," in ASRU, pp. 42-47, 2011.

[16]. C. Plahl, R. Schlÿter, and H. Ney, "Improving neural networks by preventing co-adaptation of feature detectors," CoPR, 2012.

[17]. C. Plahl et al., "Hierarchical bottle neck features for LVCSR,” Interspeech 2010, pp. 1197-1200, 2010.

[18]. A. Kurematsu et al., "ATR japanese speech database as a tool of speech recognition and synthesis," Speech Communication, No.4, pp.357-363, 1990.

[19]. X. Glorot and Y. Bengio, "Understanding the difficulty of training deep feedforward neural networks," International Conference on Artificial Intelligence and Statistics, pp. 249-256, 2010. 